PHYSICAL REVIEW D 91, 129906(E) (2015)

\title{
Erratum: Grand unified hidden-sector dark matter [Phys. Rev. D 90, 083501 (2014)]
}

Stephen J. Lonsdale and Raymond R. Volkas

(Received 6 June 2015; published 30 June 2015)

DOI: 10.1103/PhysRevD.91.129906

PACS numbers: 12.10.-g, 12.60.Jv, 12.10.Kt, 99.10.Cd

Figures 3-6 erroneously took the values of all of the dark superpartner masses to be at a common soft breaking scale despite the fact that there existed heavy dark quarks at a higher mass scale in the theory. In the updated versions of these graphs, we have corrected this mistake by requiring that, for heavy dark quarks, their superpartners are at the same common scale of those dark quarks. For the gluinos and superpartners of the light quarks below the confinement scale, their contribution to the running coupling switches off at the soft supersymmetry (SUSY) scale as before. In each of these graphs, we see minute differences in the results with a slight increase in the value of the confinement scale for each case. In the first case, we have the value of the confinement scale as a function of dark quark mass. The second scenario we consider is the variation of the confinement scale with the soft SUSY scale. In these cases we set the mass scale of the heavy dark quarks to be 100,1000 , and $10000 \mathrm{GeV}$, respectively. Again in each of these cases, we see a slight change in the value of $\Lambda$ which increases.

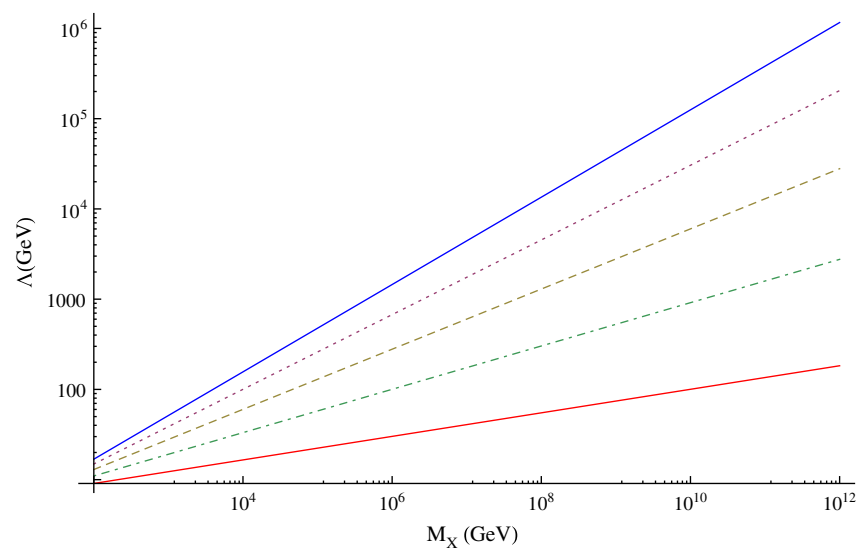

FIG. 3 (color online). Confinement scale dependence on the mass scale of heavy dark quarks $M_{X}$ with five heavy and one light quarks at the top through five light and one heavy quarks at the bottom line. All heavy quarks have mass $10000 \mathrm{GeV}$, and all light quarks are taken to have masses below $\Lambda$.

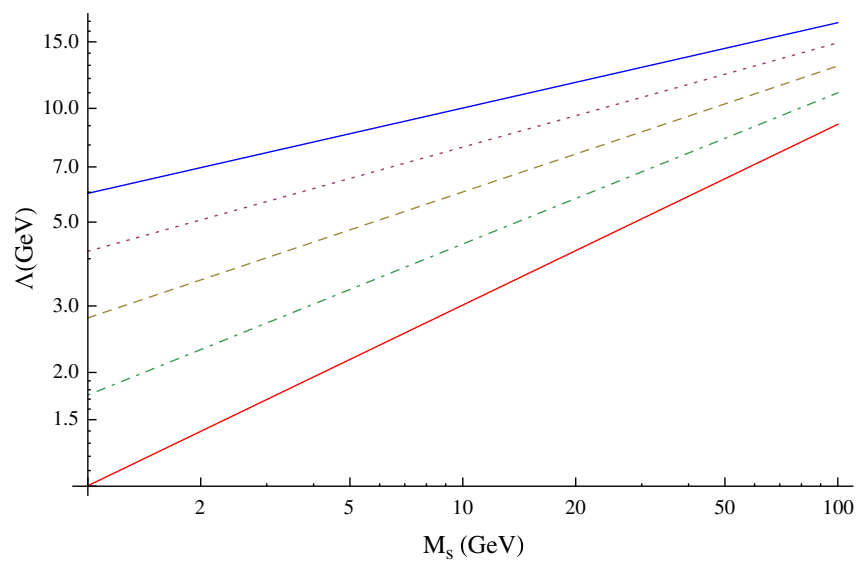

FIG. 4 (color online). Confinement scale dependence on the soft SUSY breaking scale $M_{s}$ with five heavy and one light quarks at the top through five light and one heavy quarks at the bottom line. All heavy quarks have mass $100 \mathrm{GeV}$, and all light quarks are taken to have masses below $\Lambda$. 


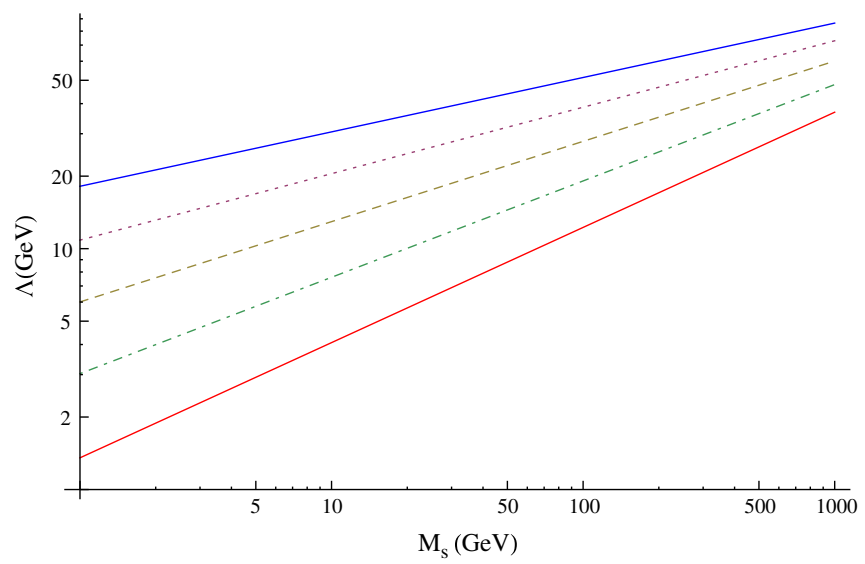

FIG. 5 (color online). Confinement scale dependence on the soft SUSY breaking scale $M_{s}$ with five heavy and one light quarks at the top through five light and one heavy quarks at the bottom line. All heavy quarks have mass $1000 \mathrm{GeV}$, and all light quarks are taken to have masses below $\Lambda$.

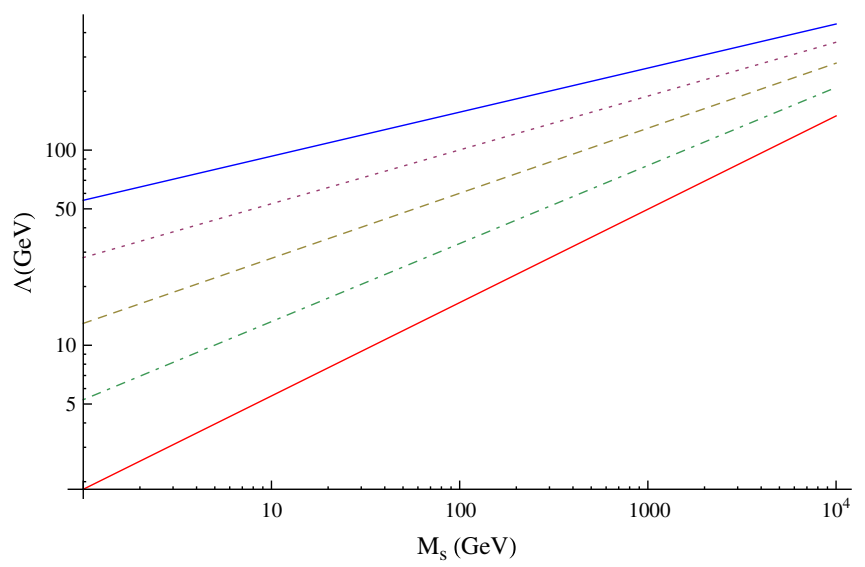

FIG. 6 (color online). Confinement scale dependence on the soft SUSY breaking scale $M_{s}$ with five heavy and one light quarks at the top through five light and one heavy quarks at the bottom line. All heavy quarks have mass $10000 \mathrm{GeV}$, and all light quarks are taken to have masses below $\Lambda$.

With these updated graphs, we can see that with only small differences in the results it remains the case that small values of the dark confinement scale close to that of the SM are still natural for large variations in the mass scales of heavy fundamental particles of a dark sector. The conclusions of the paper are therefore unchanged. 